\title{
Towards a sustainable road transportation system in urban areas: A case study of Owerri, Nigeria
}

\author{
Onyeka JO ${ }^{1}$, Obi LE ${ }^{1, *}$ and Igiri VC ${ }^{2}$ \\ ${ }^{1}$ Department of Civil Engineering, Imo State University, Owerri. \\ 2 Directorate of Works \& Services, Imo State University, Owerri.
}

International Journal of Frontiers in Engineering and Technology Research, 2021, 01(01), 014-029

Publication history: Received on 29 March 2021; revised on 30 April 2021; accepted on 02 May 2021

Article DOI: https://doi.org/10.53294/ijfetr.2021.1.1.0043

\begin{abstract}
Urban Areas in almost all cases develop outwards from the centre. Public facilities (schools, offices, markets) are usually located at about the centre while residential abodes radiate outwards. Such development pattern necessitates road transportation movement to the centre. This paper examines strategies that can be adopted to achieve the necessary movement in a sustainable manner for these urban areas, with Owerri Nigeria as a case study. A field study was conducted to ascertain the total passenger requirement, number and mix of passenger vehicles as well as measure three main road transport-induced air pollutants. The result of the field work showed existing commuter vehicles mix of 56.2:63.7: 19.6:1.6:1 for salons, wagons, mini-buses, coaster buses and big buses respectively, of a total of 85,950 vehicles. The survey also revealed ambient air pollutants level higher than the recommended standards. A new model was developed to achieve a vehicles remix of 10:33:53: 14:1 of same vehicle types and reduction in traffic volume and target air pollutants. The analyses showed that mini-buses and coaster buses have advantage over salon cars, wagon vehicles and big buses in terms of traffic congestions and pollutants release into the environment. The two bus types have least pollutants release per passenger carried. An optimal vehicle remix, which gives higher priority to these buses has been shown to reduce congestion by $40 \%$, Carbon monoxide by $40 \%$, Nitrogen Dioxide by $50 \%$ and Methane by $50 \%$. Based on the findings, it is recommended that vehicular remix of 10:33:53:14:1, for salon: wagon: mini-buses: coaster buses: big buses be adopted for Owerri commuters' transportation need. Some policy measures were put forward to help achieve this. The measures include an outright ban on use of low-passenger-carrying vehicles for commercial purposes.
\end{abstract}

Keywords: Vehicles; Mix; Sustainability; Road; Transportation

\section{Introduction}

Roads are specially prepared land ways for vehicular and pedestrian movements. In its simplest form the road can be the natural surface. It can also be in modified surface using local materials. As traffic increases the road can be expanded, and in surfaces stabilized with imported materials, which can further be surfaced to improve speed and comfort.

Transportation is the movement of humans, animals and goods from one location to another. Modes of transportation include air, land, water, cable, pipeline and space. Transportation field can be divided into infrastructure, vehicles and operations (Wikipedia, 2018). Its activities affect humans and the natural environment to a very great extent. Nevertheless, it is vital for both the development of society as a whole as well as for the mobility of individuals. The ability to transport oneself and one's products wherever and whenever necessary is seen today as a matter of expectation by the society. The design and development of the infrastructure for the transportation sector and methods

\footnotetext{
${ }^{*}$ Corresponding author: Obi LE

Directorate of Works \& Services, Imo State University, Owerri.
} 
of transportation are closely related to to general social developments and have a decisive influence on the location of housing and industry (Murtaza, H, \& Eric M., 2000). This is why coordinated efforts are necessary from actors who are active internationally, nationally, regionally and locally to ensure maximum environmental compatibility as the transport system is being transformed.

Generally and very often, the mobile units (automobiles) of the road transport system are powered by either petrol (gasoline) or diesel internal combustion engines. Such internal combustion engines are known to be major sources of outdoor air pollution, and traffic is the most notable source of air pollution in urban areas. The relentless motorization of society has entailed an increasing growth of vehicle emissions which impact negatively on urban air quality. Critical components of an integrated transportation system includes technical measures involving vehicles and fuels, transport demand management and market incentives and infrastructure \& public transport improvements. These essential components are however lacking in the transport systems of many third world countries.

Road transportation system therefore, is the aggregate of all facilities put in place, maintained and or operated for the movement of people and goods by road. These include fixed structures (roads), mobile units (motor vehicles) and the operators of these facilities.

Sustainable development is an economic development that is conducted without depletion of natural resources, OALD (2004). Generally, sustainable development implies meeting the needs of the present generation without compromising the ability of future generations to meet their own needs (Brundtland 1987). A sustainable transportation system is one in which fuel consumption, vehicle emissions, safety, congestion and social and economic access are of such levels that they can be sustained into the indefinite future without causing great or irreparable harm to future generations of people throughout the world (Richardson, 1999). This entails three dimensions namely; economic sustainability (economic efficiency); environmental sustainability (ecological stability); and social sustainability (distributional/social equity). Sustainable road transportation system is expected to meet environmental needs/standards, be economically viable and be socio-politically acceptable.

The transportation of goods and passengers is increasing world-wide. A large share of this transport can be attributed to motor vehicles which often have serious impacts on human health, environmental quality, urban development patterns, road conditions, and road safety. Increasingly, developed and developing countries are seeking strategies to guarantee individual mobility, and at the same time trying to improve ecological and social conditions. Sustainability is increasingly adopted as a framework for designing and implementing such strategies. Due to their predominant role, road transport issues are of particular concern.

The World Health Organization (WHO), while discussing Sustainability of Transport Systems, noted that clustering many passengers together in one vehicle or other public transport mode reduces total traffic emissions of climate and air pollutants. Public transport use is also associated with more physical activity and less obesity, since public transport services are often accessed by walking and cycling. The WHO further stated that investment in mass public transport can also yield equity benefits by improving the mobility of women, the elderly and the poor, who often lack access to private vehicles. This, in turn, provides employment, education, health services and recreational opportunities (WHO, 2020).

Writing on Sustainable Transportation Options for Protecting the Climate, Drum-heller et al, (2001), noted that the sequence of actions leading to the creation of a transport system can be as follows;

- Analysis of the existing transport system;

- Determination of requirements for the new transport system;

- Selection of transport combination

The analysis of the existing system is the starting point for actions aiming at an improvement of the current situation. The analysis must include the current flows in the transport system, future demand for carriage of passengers and goods and the consequences of the functioning of the current system such as road congestion; noise emission; toxic emissions; accident statistics. In the next step is the need to identify the objective that to be achieved e.g. by determining the level of admissible exhaust emissions at a given flow density of vehicles.

The selection of the transport combination covers the identification of admissible solutions for example such related to the modification of the traffic organization (new traffic nodes, modification of the traffic signaling, limits in vehicle flow of a given type roads) and the evaluation of the consequences of their implementation. At the final stage of the process 
a decision is made as to which solution is the best in terms of the decision maker expectations, i.e. maximizes the function of benefit at assumed limitations (for example financial).

Merkisz-Guranoroska et al, (2013), in their work on Development of a Sustainable Road Transport System added that particularly difficult is the development of transport systems where the fundamental requirements for the new systems are environment related. Taking the environmental aspects into account limits the development of road transport systems that somewhat generate negative environmental impacts. The priority of the investors and decision makers is most often the maximization of the throughput of the new system at given limitations (related to the investment expenditure) or, possibly, building infrastructure at a minimum cost for a given network efficiency. Reducing negative impact of transportation on the environment either increases the capital expenditure for the construction of the system or limits the traffic, thus the system throughput. The complexity of such a decision problem requires a development of a decision-support tool for the best decision in terms of the configuration of a transport system meeting the assumed traffic throughput requirements but taking the environmental aspects into account at an acceptable level of capital expenditure. The decision-support tool in the said area may constitute a model of development of a pro-ecological transport system.

Modeling plays an important role in the cognitive process enabling the exploration of the relations and processes in complex systems. Besides, modeling enables a simulation of the functioning of a system depending on the implemented modifications and system organization. The effect of the model implementation will be to create the possibility of determination of the influence of transport node solutions and traffic organization of the actual level of exhaust emission in road transport. The application of the model will lead to conclusions as to how the structure of the road transport flow in connection with the road transport infrastructure influence the environment pollution level.

One sure way of arriving at the best sustainable road transportation system is by the optimization of such models.

Optimization is the act of obtaining the best solution under given circumstances. This technique provides a powerful tool in improving the engineering design in a rational manner and has been proved to be much more efficient than the traditional trial-and-error design process (Aravelli, 2014). Today, the optimization tool has become a part of every engineering study for design improvement.

Urban area is a human settlement with a high population density and infrastructure of built environment, (Wikipedia, 2020). Urban Areas in almost all cases develop outwards from the centre. Public facilities (schools, offices, markets) are usually located at about the centre while residential abodes radiate outwards. Such development pattern necessitates road transportation movement to the centre

Owerri, the capital city of Imo State of Nigeria, is growing outwards from the city centre just as every other urban area. It sits at the intersection of roads from Port Harcourt, Onitsha, Aba, Umuahia, Okigwe and Orlu and at latitude $5^{\circ} 28^{\prime}$ $35.6^{\prime \prime}\left(5.4766^{\circ}\right)$ North and longitudes 7o $1^{\prime} 0.6^{\prime \prime}\left(7.0168^{\circ}\right)$ East and elevation of $75 \mathrm{~m}$ above sea level Encyclopaedia Britanica (2009).

\section{Material and methods}

The mobility need of commuters result in the use of vehicles and the powering of the vehicles produces air pollutants and could result in congestion on the roads, thereby hindering movement. The method of assessment therefore includes;

- Determination of the transport need and

- Measurement of pollution level (pollutants of interest)

- Determination of congestion level (Level of Service)

The assessment was carried out at five strategic representative locations in the Owerri Municipal viz; Amakohia Junction, Assumpta Junction, Emmanuel College Junction, Imo State University Junction and MCC/Wethedral Road Junction. The selected locations for the survey are points with high traffic and business activities. The result of the assessment was imputed into a model in terms of vehicle types and exhaust emissions, and optimized for vehicle and emission reductions.

\subsection{Determination of Transport Need/Load}

The vehicles, (in their various classes), traversing the five locations in the project area were counted over a 12-hour period - 7am to 6p.m. Based on an observed percentage occupancy of the passenger vehicles, the total passenger 
transportation needs at the five locations was determined. These locations record high traffic volumes within the hours of 7.30 - 9.30am (when offices and commercial activities commence) and $4.00-7.00 \mathrm{pm}$ in the evening at the close of work and market activities.

The time-segmented transportation needs/loads as well as the cumulative transportation needs/loads at the locations was evaluated.

\subsection{Measurement of Target Air Pollutants}

The target air pollutants - carbon monoxide (CO), Nitrogen dioxide ( $\mathrm{NO}_{2}$ ), Hydrocarbons-Methane (CH4) were measured using standard equipment called Aeroqual/crowcon Gasman Monitors. The measurements were at three intervals within 12 hours in a day for a total of two days at each location.

\section{Results and Analysis}

The field survey results obtained at the different locations were subsequently analyzed.

\subsection{Survey Results}

The vehicle counts, in types, from the four approaches, for each survey day are collated and the cumulative figures as well as the corresponding measured pollutants are presented in tables.

\subsection{IMSU Junction}

The figures for this junction are presented in Table 1 for day 1 and Table 2 for day 2 .

Table 1 vehicle types for day 1

\begin{tabular}{|c|c|c|c|c|c|c|c|c|c|}
\hline \multirow[t]{2}{*}{ Period } & \multicolumn{6}{|c|}{ Vehicle Types } & \multicolumn{3}{|c|}{ Pollutants } \\
\hline & $\begin{array}{l}\text { Sal. Veh. } \\
V_{1}\end{array}$ & $\begin{array}{l}\text { Wagon } \\
\text { Veh. V } 2 \\
\end{array}$ & $\begin{array}{l}\text { Mini } \\
\text { Buses V }\end{array}$ & $\begin{array}{l}\text { Coaster } \\
\text { Buses V } 4\end{array}$ & $\begin{array}{l}\text { Big } \\
\text { Buses V } \\
\end{array}$ & $\begin{array}{l}\text { Trucks } \\
\mathrm{V}_{6}\end{array}$ & $\begin{array}{l}\text { CO } \\
\text { ppm }\end{array}$ & $\begin{array}{l}\mathrm{NO}_{2} \\
\mathrm{Ppm}\end{array}$ & $\begin{array}{l}\mathrm{CH}_{4} \\
\mathrm{Ppm} \\
\end{array}$ \\
\hline $\begin{array}{l}\text { Morning } \\
\text { (At 7am) }\end{array}$ & 847 & 833 & 186 & 37 & 22 & 262 & 09 & 18 & 0.031 \\
\hline $\begin{array}{l}\text { Afternoon } \\
\text { (At 2pm) }\end{array}$ & 10,112 & 8261 & 2628 & 125 & 54 & 684 & 05 & 0.169 & ND \\
\hline \multirow[t]{2}{*}{$\begin{array}{l}\text { Evening } \\
\text { (At 6pm) }\end{array}$} & 14,579 & 12,487 & 4045 & 201 & 79 & 944 & 05 & 0.090 & ND \\
\hline & \multicolumn{6}{|c|}{ Standards } & 10 & $0.04-0.06$ & NS \\
\hline
\end{tabular}

Table 2 Vehicle types for day 2

\begin{tabular}{|c|c|c|c|c|c|c|c|c|c|}
\hline \multirow[t]{2}{*}{ Period } & \multicolumn{6}{|c|}{ Vehicle Types } & \multicolumn{3}{|c|}{ Pollutants } \\
\hline & $\begin{array}{c}\text { Sal. Veh. } \\
\qquad V_{1}\end{array}$ & $\begin{array}{l}\text { Wagon } \\
\text { Veh. V } \\
\end{array}$ & $\begin{array}{c}\text { Mini } \\
\text { Buses } V_{3}\end{array}$ & $\begin{array}{c}\text { Coaster } \\
\text { Buses V }\end{array}$ & $\begin{array}{c}\text { Big Buses } \\
V_{5}\end{array}$ & $\begin{array}{c}\text { Trucks } \\
\mathrm{V}_{6} \\
\end{array}$ & $\begin{array}{c}\text { CO } \\
\text { ppm }\end{array}$ & $\begin{array}{l}\mathrm{NO}_{2} \\
\mathrm{Ppm}\end{array}$ & $\begin{array}{l}\mathrm{CH}_{4} \\
\mathrm{Ppm} \\
\end{array}$ \\
\hline $\begin{array}{l}\text { Morning } \\
\text { (At 7am) }\end{array}$ & 687 & 769 & 112 & 09 & 04 & 34 & 09 & 0.084 & ND \\
\hline $\begin{array}{l}\text { Afternoon } \\
\text { (At 2pm) }\end{array}$ & 15090 & 12661 & 2343 & 229 & 78 & 297 & 06 & 0.040 & ND \\
\hline $\begin{array}{l}\text { Evening } \\
\text { (At 6pm) }\end{array}$ & 25022 & 20813 & 3443 & 357 & 106 & 555 & 14 & 0.086 & ND \\
\hline & \multicolumn{6}{|c|}{ Standards } & 10 & 0.04-0.06 & NS \\
\hline
\end{tabular}


At this junction one of the measured values of $\mathrm{CO}$ is above the standard, two values are close to the standard while three are below the standard. Five of the Nitrogen dioxide values are above the standard while one is within the standard. Methane was only detected on one out of the six times. There is also a high volume of salon and wagon vehicles traversing this junction throughout the survey period, and occasionally resulting in traffic jam.

\subsection{Amakohia Junction}

The corresponding figures for Amakohia Junction are presented in Tables 3.3 and 3.4.

Table 3 Vehicle types day 1

\begin{tabular}{|c|c|c|c|c|c|c|c|c|c|}
\hline \multirow[t]{2}{*}{ Period } & \multicolumn{6}{|c|}{ Vehicle Types } & \multicolumn{3}{|c|}{ Pollutants } \\
\hline & $\begin{array}{c}\text { Sal. } \\
\text { Veh. } \\
\text { V }_{1}\end{array}$ & $\begin{array}{c}\text { Wago } \\
\text { n Veh. } \\
V_{2}\end{array}$ & $\begin{array}{c}\text { Mini } \\
\text { Buses } \\
\mathbf{V}_{3} \\
\end{array}$ & $\begin{array}{c}\text { Coaster } \\
\text { Buses } \\
\mathbf{V}_{4} \\
\end{array}$ & $\begin{array}{c}\text { Big } \\
\text { Buses } \\
\text { V }_{5} \\
\end{array}$ & $\begin{array}{c}\text { Truck } \\
\text { S } \\
\mathrm{V}_{6} \\
\end{array}$ & $\begin{array}{c}\text { CO } \\
\text { ppm }\end{array}$ & $\begin{array}{l}\mathrm{NO}_{2} \\
\mathrm{Ppm}\end{array}$ & $\begin{array}{c}\mathrm{CH}_{4} \\
\mathbf{P p} \\
\mathbf{m}\end{array}$ \\
\hline $\begin{array}{l}\text { Morning } \\
\text { (At 7am) }\end{array}$ & 754 & 597 & 160 & 08 & 05 & 57 & 3 & 0.134 & ND \\
\hline $\begin{array}{l}\text { Afternoon } \\
\text { (At 2pm) }\end{array}$ & 7628 & 7110 & 1435 & 121 & 70 & 402 & 3 & 0.073 & ND \\
\hline $\begin{array}{l}\text { Evening } \\
\text { (At 6pm) }\end{array}$ & 14057 & 13831 & 3285 & 297 & 127 & 783 & 7 & 0.111 & ND \\
\hline & \multicolumn{6}{|c|}{ Standards } & 10 & 0.04-0.06 & NS \\
\hline
\end{tabular}

Table 4 Vehicle types 2

\begin{tabular}{|c|c|c|c|c|c|c|c|c|c|}
\hline \multirow[t]{2}{*}{ Period } & \multicolumn{6}{|c|}{ Vehicle Types } & \multicolumn{3}{|c|}{ Pollutants } \\
\hline & $\begin{array}{l}\text { Sal. Veh. } \\
V_{1}\end{array}$ & $\begin{array}{l}\text { Wagon } \\
\text { Veh. } V_{2} \\
\end{array}$ & $\begin{array}{l}\text { Mini } \\
\text { Buses V } 3 \\
\end{array}$ & $\begin{array}{l}\text { Coaster } \\
\text { Buses V } 4 \\
\end{array}$ & $\begin{array}{l}\text { Big Buses } \\
V_{5}\end{array}$ & $\begin{array}{l}\text { Trucks } \\
\mathrm{V}_{6}\end{array}$ & \begin{tabular}{|l|} 
CO \\
ppm \\
\end{tabular} & $\begin{array}{l}\mathrm{NO}_{2} \\
\mathrm{Ppm} \\
\end{array}$ & $\begin{array}{l}\mathrm{CH}_{4} \\
\mathrm{Ppm} \\
\end{array}$ \\
\hline $\begin{array}{l}\text { Morning } \\
\text { (At 7am) }\end{array}$ & 607 & 601 & 63 & 05 & 02 & 42 & 7 & 0.06 & ND \\
\hline $\begin{array}{l}\text { Afternoon } \\
\text { (At 2pm) }\end{array}$ & 10581 & 8187 & 3405 & 544 & 62 & 435 & 6 & 0.043 & ND \\
\hline $\begin{array}{l}\text { Evening } \\
\text { (At 6pm) }\end{array}$ & 16218 & 15311 & 5526 & 645 & 120 & 806 & 8 & 0.073 & ND \\
\hline & \multicolumn{6}{|l|}{ Standards } & 10 & $0.04-0.06$ & NS \\
\hline
\end{tabular}

ND = NONE DETECTED; NS = NOT STATED

Three of the Carbon monoxide values at this junction are well below the standard while three are very close to it. Four of the nitrogen dioxide values are well above the standard while two are within the standard. Methane was not detected.

There is also a high volume of salons and wagons at the junction, in excess of five times the total of other passenger vehicles.

\subsection{Assumpta Junction}

The corresponding figures for Assumpta Junction are presented in tables 3.5 and 3.6.

Two of the Carbon monoxide values recorded at this junction are above the standard while four values are below the standard. Four values of nitrogen-dioxide are above standards while two are within the standards. Only on one occasion was a value recorded for methane.

The salons and wagons traversing this junction are close to ten times that of other passenger vehicles, very often resulting in traffic gridlock. 
Table 5 Vehicle types for day 1

\begin{tabular}{|c|c|c|c|c|c|c|c|c|c|}
\hline \multirow[t]{2}{*}{ Period } & \multicolumn{6}{|c|}{ Vehicle Types } & \multicolumn{3}{|c|}{ Pollutants } \\
\hline & $\begin{array}{l}\text { Sal. } \\
\text { Veh. } V_{1}\end{array}$ & $\begin{array}{l}\text { Wagon } \\
\text { Veh. } V_{2}\end{array}$ & $\begin{array}{c}\text { Mini } \\
\text { Buses } V_{3}\end{array}$ & $\begin{array}{c}\text { Coaster } \\
\text { Buses } V_{4}\end{array}$ & $\begin{array}{c}\text { Big } \\
\text { Buses } V_{5}\end{array}$ & $\begin{array}{c}\text { Trucks } \\
V_{6}\end{array}$ & $\begin{array}{c}\text { CO } \\
\text { Ppm }\end{array}$ & $\begin{array}{l}\mathrm{NO}_{2} \\
\mathrm{Ppm}\end{array}$ & $\begin{array}{l}\mathrm{CH}_{4} \\
\mathrm{Ppm}\end{array}$ \\
\hline $\begin{array}{l}\text { Morning } \\
\text { (At 7am) }\end{array}$ & 743 & 874 & 215 & 13 & 15 & 69 & ND & 0.090 & 1.00 \\
\hline $\begin{array}{l}\text { Afternoon } \\
\text { (At 2pm) }\end{array}$ & 11332 & 10304 & 2152 & 135 & 74 & 714 & 6.00 & 0.060 & ND \\
\hline Evening & 20429 & 16775 & 3637 & 215 & 119 & 1727 & 17.00 & 0.005 & ND \\
\hline & \multicolumn{6}{|c|}{ Standards } & 10 & $0.04-0.06$ & NS \\
\hline
\end{tabular}

Table 6 Vehicle types for day 2

\begin{tabular}{|c|c|c|c|c|c|c|c|c|c|}
\hline \multirow[t]{2}{*}{ Period } & \multicolumn{6}{|c|}{\begin{tabular}{|l} 
Vehicle Types \\
\end{tabular}} & \multicolumn{3}{|c|}{ Pollutants } \\
\hline & $\begin{array}{ll}\text { Sal. } & \text { Veh. } \\
\text { V}_{1} & \end{array}$ & $\begin{array}{l}\text { Wagon } \\
\text { Veh. } V_{2}\end{array}$ & \begin{tabular}{|l} 
Mini \\
Buses V
\end{tabular} & \begin{tabular}{|l} 
Coaster \\
Buses V $_{4}$
\end{tabular} & \begin{tabular}{|l} 
Big Buses \\
$V_{5}$
\end{tabular} & \begin{tabular}{|l} 
Trucks \\
$\mathrm{V}_{6}$
\end{tabular} & $\begin{array}{l}\text { CO } \\
\text { Ppm }\end{array}$ & $\begin{array}{l}\mathrm{NO}_{2} \\
\mathrm{Ppm}\end{array}$ & $\begin{array}{l}\mathrm{CH}_{4} \\
\mathrm{Ppm}\end{array}$ \\
\hline $\begin{array}{l}\text { Morning } \\
\text { (At 7am) }\end{array}$ & 715 & 586 & 229 & 15 & 22 & 75 & 3.00 & 0.086 & ND \\
\hline $\begin{array}{l}\text { Afternoon (At } \\
2 \mathrm{pm})\end{array}$ & 9733 & 11291 & 2908 & 275 & 234 & 507 & 11.00 & 087 & ND \\
\hline \multirow[t]{2}{*}{$\begin{array}{l}\text { Evening (At } \\
6 \mathrm{pm})\end{array}$} & 14534 & 18853 & 3819 & 382 & 357 & 835 & 3.00 & 0.137 & ND \\
\hline & \multicolumn{6}{|c|}{ Standards } & 10 & 0.04-0.06 & NS \\
\hline
\end{tabular}

ND: NON-DETECTED NS: NOT STATED

\subsection{Emmanuel College}

The corresponding figures for Emmanuel College junction are presented in Tables 3.7 and 3.8.

Table 7 Vehicle types for day 1

\begin{tabular}{|c|c|c|c|c|c|c|c|c|c|}
\hline \multirow[t]{2}{*}{ Period } & \multicolumn{6}{|c|}{ Vehicle Types } & \multicolumn{3}{|c|}{ Pollutants } \\
\hline & Sal. Veh. $V_{1}$ & $\begin{array}{l}\text { Wagon } \\
\text { Veh. } V_{2}\end{array}$ & $\begin{array}{c}\text { Mini } \\
\text { Buses } V_{3}\end{array}$ & $\begin{array}{c}\text { Coaster } \\
\text { Buses } V_{4}\end{array}$ & $\begin{array}{c}\text { Big } \\
\text { Buses } V_{5}\end{array}$ & $\begin{array}{c}\text { Trucks } \\
V_{6}\end{array}$ & $\begin{array}{c}\text { CO } \\
\text { Ppm }\end{array}$ & $\begin{array}{l}\mathrm{NO}_{2} \\
\mathrm{Ppm}\end{array}$ & $\begin{array}{c}\mathrm{CH}_{4} \\
\mathrm{Ppm}\end{array}$ \\
\hline $\begin{array}{l}\text { Morning } \\
\text { (At 7am) }\end{array}$ & 1498 & 2816 & 527 & 89 & 60 & 158 & 31.00 & 0.070 & ND \\
\hline $\begin{array}{l}\text { Afternoon } \\
\text { (At 2pm) }\end{array}$ & 11154 & 11635 & 5014 & 473 & 316 & 584 & 17.00 & 0.048 & ND \\
\hline $\begin{array}{l}\text { Evening } \\
\text { (At 6pm) }\end{array}$ & 21184 & 20391 & 11921 & 953 & 607 & 1276 & 7.00 & 0.068 & 1.00 \\
\hline & \multicolumn{6}{|c|}{ Standards } & 10 & $0.04-0.06$ & NS \\
\hline
\end{tabular}

ND = NONE DETECTED NS = NOT STATED 
Table 8 Vehicle types for day 2

\begin{tabular}{|c|c|c|c|c|c|c|c|c|c|}
\hline \multirow[t]{2}{*}{ Period } & \multicolumn{6}{|c|}{ Vehicle Types } & \multicolumn{3}{|c|}{ Pollutants } \\
\hline & $\begin{array}{l}\text { Sal. Veh. } \\
V_{1}\end{array}$ & $\begin{array}{l}\text { Wagon } \\
\text { Veh. } V_{2}\end{array}$ & $\begin{array}{l}\text { Mini } \\
\text { Buses } V_{3}\end{array}$ & $\begin{array}{l}\text { Coaster } \\
\text { Buses V } 4\end{array}$ & $\begin{array}{l}\text { Big } \\
\text { Buses } V_{5}\end{array}$ & $\begin{array}{l}\text { Trucks } \\
V_{6}\end{array}$ & $\begin{array}{l}\text { CO } \\
\text { Ppm }\end{array}$ & $\begin{array}{l}\mathrm{NO}_{2} \\
\mathrm{Ppm}\end{array}$ & $\begin{array}{l}\mathrm{CH}_{4} \\
\mathrm{Ppm}\end{array}$ \\
\hline $\begin{array}{l}\text { Morning } \\
\text { (At 7am) }\end{array}$ & 876 & 2135 & 434 & 38 & 33 & 180 & 80.00 & 0.073 & 1.00 \\
\hline $\begin{array}{l}\text { Afternoon } \\
\text { (At 2pm) }\end{array}$ & 27734 & 32567 & 6706 & 114 & 128 & 1318 & 22.00 & 0.092 & ND \\
\hline Evening & 34101 & 38368 & 9496 & 228 & 227 & 1697 & 13.00 & 0.182 & ND \\
\hline & \multicolumn{6}{|l|}{ Standards } & 10 & $0.04-0.06$ & NS \\
\hline
\end{tabular}

ND $=$ NONE DETECTED NS = NOT STATED

All the values of the Carbon-monoxide measured here are above the standard and in most cases about double the standard. All but one nitrogen dioxide values are above the standard while methane was detected on two out of the six times. There is a very high volume of salons and wagons at this junction, over eight times the total of other passenger vehicles. Expectedly traffic jam is very often experienced at the junction.

\subsection{Wethedral/MCC Junction}

The corresponding figures for the Wethedral/MCC junction are presented in Tables 3.9 and 3.10.

Table 9 Vehicle types for day 1

\begin{tabular}{|c|c|c|c|c|c|c|c|c|c|}
\hline \multirow[t]{2}{*}{ Period } & \multicolumn{6}{|c|}{ Vehicle Types } & \multicolumn{3}{|c|}{ Pollutants } \\
\hline & $\begin{array}{c}\text { Sal. Veh. } \\
\quad V_{1}\end{array}$ & $\begin{array}{l}\text { Wagon } \\
\text { Veh. } V_{2}\end{array}$ & $\begin{array}{c}\text { Mini } \\
\text { Buses } V_{3}\end{array}$ & $\begin{array}{c}\text { Coaster } \\
\text { Buses } V_{4}\end{array}$ & $\begin{array}{c}\text { Big } \\
\text { Buses } V_{5}\end{array}$ & $\begin{array}{c}\text { Trucks } \\
V_{6}\end{array}$ & $\begin{array}{c}\text { CO } \\
\text { Ppm }\end{array}$ & $\begin{array}{l}\mathrm{NO}_{2} \\
\mathrm{Ppm}\end{array}$ & $\begin{array}{l}\mathrm{CH}_{4} \\
\mathrm{Ppm}\end{array}$ \\
\hline $\begin{array}{l}\text { Morning } \\
\text { (At 7am) }\end{array}$ & 1108 & 2353 & 112 & 18 & 24 & 86 & 20.00 & 0.072 & ND \\
\hline $\begin{array}{l}\text { Afternoon } \\
\text { (At 2pm) }\end{array}$ & 16151 & 15702 & 389 & 62 & 85 & 316 & 13.00 & 0.084 & ND \\
\hline $\begin{array}{l}\text { Evening } \\
\text { (At 6pm) }\end{array}$ & 30647 & 23098 & 808 & 164 & 195 & 633 & 21.00 & 0.126 & ND \\
\hline & \multicolumn{6}{|c|}{ Standards } & 10 & $0.04-0.06$ & NS \\
\hline
\end{tabular}

Table 10 Cumulative vehicle types for day 2

\begin{tabular}{|c|c|c|c|c|c|c|c|c|c|}
\hline \multirow[t]{2}{*}{ Period } & \multicolumn{6}{|c|}{ Cumulative Vehicle Types } & \multicolumn{3}{|c|}{ Pollutants } \\
\hline & $\begin{array}{c}\text { Sal. Veh. } \\
\mathbf{V}_{\mathbf{1}}\end{array}$ & $\begin{array}{l}\text { Wagon } \\
\text { Veh. } V_{2}\end{array}$ & $\begin{array}{c}\text { Mini } \\
\text { Buses } V_{3}\end{array}$ & $\begin{array}{c}\text { Coaster } \\
\text { Buses } V_{4}\end{array}$ & $\begin{array}{c}\text { Big } \\
\text { Buses } V_{5}\end{array}$ & $\begin{array}{c}\text { Trucks } \\
V_{6}\end{array}$ & $\begin{array}{c}\text { CO } \\
\text { Ppm }\end{array}$ & $\begin{array}{l}\mathrm{NO}_{2} \\
\mathrm{Ppm}\end{array}$ & $\begin{array}{c}\mathrm{CH}_{4} \\
\mathrm{Ppm}\end{array}$ \\
\hline $\begin{array}{l}\text { Morning } \\
\text { (At 7am) }\end{array}$ & 383 & 734 & 45 & 04 & 06 & 35 & 22.00 & 0.075 & ND \\
\hline $\begin{array}{l}\text { Afternoon } \\
\text { (At 2pm) }\end{array}$ & 6639 & 13537 & 579 & 61 & 76 & 254 & 17.00 & ND & ND \\
\hline \multirow[t]{2}{*}{$\begin{array}{l}\text { Evening (At } \\
6 \mathrm{pm} \text { ) }\end{array}$} & 10509 & 19579 & 883 & 106 & 107 & 405 & 3.00 & 0.091 & ND \\
\hline & \multicolumn{6}{|c|}{ Standards } & 10 & $0.04-0.06$ & NS \\
\hline
\end{tabular}


At this junction all but one value of Carbon monoxide is well above the standard. On five of the six times the nitrogen dioxide values are above the standard while methane was not detected. There is a very high volume of salons and wagons at the junction, in excess of over forty times that of other passenger vehicles, on the average, with traffic gridlock very often.

\subsection{Exhaust Emission of vehicles.}

The representative vehicles exhaust emissions for the three pollutants of interest are presented on Table 11.

Table 11 Exhaust Emission of Different Vehicles

\begin{tabular}{|c|c|c|c|}
\hline \multirow[t]{2}{*}{ Vehicle } & \multicolumn{3}{|c|}{ Pollutants } \\
\hline & $\begin{array}{c}\text { Co, } \\
\text { ppm }\end{array}$ & $\begin{array}{l}\mathrm{NO}_{2}, \\
\text { ppm }\end{array}$ & $\begin{array}{l}\mathrm{CH}_{4}, \\
\text { ppm }\end{array}$ \\
\hline Car 1 & 234.30 & 0.56 & 284.00 \\
\hline Car 2 & 234.30 & 0.30 & 5613.00 \\
\hline Car 3 & 234.30 & 0.60 & 4227.00 \\
\hline Average & 234.30 & 0.49 & 4229.00 \\
\hline Wagon 1 & 173.80 & 0.93 & 79.00 \\
\hline Wagon 2 & 68.80 & 0.49 & 87.00 \\
\hline Wagon 3 & 234.30 & 0.04 & 79.00 \\
\hline Average & 158.97 & 0.49 & 81.67 \\
\hline Mini Bus 1 & 234.30 & 0.28 & 3797.00 \\
\hline Mini Bus 2 & 234.30 & 0.22 & 4461.00 \\
\hline Mini Bus 3 & 209.20 & 0.15 & 512.00 \\
\hline Average & 225.93 & 0.22 & 2923.33 \\
\hline Coaster Bus 1 & 234.30 & 0.63 & 479.00 \\
\hline Coaster Bus 2 & 234.30 & 0.63 & 444.00 \\
\hline Coaster Bus 3 & 234.30 & 0.63 & 396.00 \\
\hline Average & 234.30 & 0.63 & 439.67 \\
\hline Big Bus 1 & ND & 5.37 & 14.00 \\
\hline Big Bus 2 & ND & 5.37 & 14.00 \\
\hline Big Bus 3 & ND & 5.37 & 10.00 \\
\hline Average & ND & 5.37 & 12.67 \\
\hline Truck 1 & 254.60 & 1.03 & 18.00 \\
\hline Truck 2 & 246.80 & 1.26 & 16.00 \\
\hline Truck 3 & 254.60 & 1.27 & 20.00 \\
\hline Average & 252.00 & 1.19 & 18.00 \\
\hline
\end{tabular}

\subsection{Analysis of Results}

Applying the principle of worst case scenario on the collated results and measured pollutants for the survey locations yields the following Tables. 


\subsection{Worst Case Results}

The worst case results for the five junctions are presented in Tables 3.12, 3.13, 3.14, 3.15 and 3.16.

Table 12 For IMSU junction

\begin{tabular}{|c|c|c|c|c|c|c|c|c|c|}
\hline \multirow[t]{2}{*}{ Period } & \multicolumn{6}{|c|}{ Cumulative Vehicle Types } & \multicolumn{3}{|c|}{ Pollutants } \\
\hline & $\begin{array}{l}\text { Sal. Veh. } \\
V_{1}\end{array}$ & $\begin{array}{l}\text { Wagon } \\
\text { Veh. V }\end{array}$ & $\begin{array}{l}\text { Mini } \\
\text { Buses V } 3\end{array}$ & $\begin{array}{l}\text { Coaster } \\
\text { Buses V4 }\end{array}$ & $\begin{array}{l}\text { Big } \\
\text { Buses V5 }\end{array}$ & $\begin{array}{l}\text { Trucks } \\
V_{6}\end{array}$ & $\begin{array}{l}\text { CO } \\
\text { Ppm }\end{array}$ & $\begin{array}{l}\mathrm{NO}_{2} \\
\mathrm{ppm}\end{array}$ & $\begin{array}{l}\mathrm{CH}_{4} \\
\mathrm{Ppm}\end{array}$ \\
\hline $\begin{array}{l}\text { Morning } \\
\text { (At 7am) }\end{array}$ & 847 & 833 & 186 & 37 & 22 & 262 & 09 & 18 & 0.031 \\
\hline $\begin{array}{l}\text { Afternoon } \\
\text { (At 2pm) }\end{array}$ & 15090 & 12661 & 3403 & 229 & 78 & 684 & 06 & 0.169 & ND \\
\hline $\begin{array}{l}\text { Evening } \\
\text { (At 6pm) }\end{array}$ & 25022 & 20813 & 4820 & 357 & 106 & 944 & 14 & 0.090 & ND \\
\hline
\end{tabular}

Table 13 For amakohia junction

\begin{tabular}{|c|c|c|c|c|c|c|c|c|c|}
\hline \multirow[t]{2}{*}{ Period } & \multicolumn{6}{|c|}{ Cumulative Vehicle Types } & \multicolumn{3}{|c|}{ Pollutants } \\
\hline & $\begin{array}{c}\text { Sal. } \\
\text { Veh. V1 }\end{array}$ & $\begin{array}{l}\text { Wagon Veh. } \\
\mathrm{V}_{2}\end{array}$ & $\begin{array}{c}\text { Mini } \\
\text { Buses V3 }\end{array}$ & $\begin{array}{l}\text { Coaster } \\
\text { Buses V4 }\end{array}$ & $\begin{array}{c}\text { Big } \\
\text { Buses V5 }\end{array}$ & $\begin{array}{c}\text { Trucks } \\
\mathrm{V}_{6}\end{array}$ & $\begin{array}{c}\text { CO } \\
\text { Ppm }\end{array}$ & $\begin{array}{l}\mathrm{NO}_{2} \\
\text { ppm }\end{array}$ & $\begin{array}{r}\mathrm{CH}_{4} \\
\mathrm{Ppm}\end{array}$ \\
\hline $\begin{array}{l}\text { Morning } \\
\text { (At 7am) }\end{array}$ & 754 & 601 & 160 & 08 & 05 & 57 & 7 & 0.134 & ND \\
\hline $\begin{array}{l}\text { Afternoon } \\
\text { (At 2pm) }\end{array}$ & 10581 & 8187 & 3405 & 544 & 70 & 435 & 6 & 0.073 & ND \\
\hline $\begin{array}{l}\text { Evening } \\
\text { (At 6pm) }\end{array}$ & 16218 & 15311 & 5526 & 645 & 127 & 806 & 8 & 0.111 & ND \\
\hline
\end{tabular}

Table 14 For amakohia junction

\begin{tabular}{|c|c|c|c|c|c|c|c|c|c|}
\hline \multirow[t]{2}{*}{ Period } & \multicolumn{6}{|c|}{ Cumulative Vehicle Types } & \multicolumn{3}{|c|}{ Pollutants } \\
\hline & $\begin{array}{c}\text { Sal. Veh. } \\
\qquad V_{1}\end{array}$ & $\begin{array}{l}\text { Wagon } \\
\text { Veh. V } 2\end{array}$ & $\begin{array}{c}\text { Mini } \\
\text { Buses } V_{3}\end{array}$ & $\begin{array}{c}\text { Coaster } \\
\text { Buses V4 }\end{array}$ & $\begin{array}{c}\text { Big } \\
\text { Buses V }\end{array}$ & $\begin{array}{c}\text { Trucks } \\
\mathrm{V}_{6}\end{array}$ & $\begin{array}{c}\text { CO } \\
\text { Ppm }\end{array}$ & $\begin{array}{l}\mathrm{NO}_{2} \\
\text { ppm }\end{array}$ & $\begin{array}{l}\mathrm{CH}_{4} \\
\mathrm{Ppm}\end{array}$ \\
\hline $\begin{array}{l}\text { Morning } \\
\text { (At 7am) }\end{array}$ & 743 & 874 & 226 & 15 & 22 & 75 & 3.00 & 0.090 & 1.00 \\
\hline $\begin{array}{l}\text { Afternoon } \\
\text { (At 2pm) }\end{array}$ & 11332 & 11291 & 2908 & 275 & 234 & 714 & 11.00 & 0.087 & ND \\
\hline $\begin{array}{l}\text { Evening } \\
\text { (At 6pm) }\end{array}$ & 20429 & 18853 & 3819 & 382 & 357 & 1727 & 17.00 & 0.137 & ND \\
\hline
\end{tabular}


Table 15 For emmanuel college junction

\begin{tabular}{|c|c|c|c|c|c|c|c|c|c|}
\hline \multirow[t]{2}{*}{ Period } & \multicolumn{6}{|c|}{ Cumulative Vehicle Types } & \multicolumn{3}{|c|}{ Pollutants } \\
\hline & $\begin{array}{c}\text { Sal. Veh. } \\
\quad V_{1}\end{array}$ & $\begin{array}{l}\text { Wagon } \\
\text { Veh. } V_{2}\end{array}$ & $\begin{array}{c}\text { Mini } \\
\text { Buses } V_{3}\end{array}$ & $\begin{array}{c}\text { Coaster } \\
\text { Buses } V_{4}\end{array}$ & $\begin{array}{c}\text { Big } \\
\text { Buses } V_{5}\end{array}$ & $\begin{array}{c}\text { Trucks } \\
V_{6}\end{array}$ & $\begin{array}{c}\text { CO } \\
\text { Ppm }\end{array}$ & $\begin{array}{l}\mathrm{NO}_{2} \\
\mathrm{Ppm}\end{array}$ & $\begin{array}{l}\mathrm{CH}_{4} \\
\mathrm{Ppm}\end{array}$ \\
\hline $\begin{array}{l}\text { Morning } \\
\text { (At 7am) }\end{array}$ & 1498 & 2816 & 527 & 89 & 60 & 180 & 80.00 & 0.073 & 1.00 \\
\hline $\begin{array}{l}\text { Afternoon } \\
\text { (At 2pm) }\end{array}$ & 11154 & 11635 & 6706 & 473 & 316 & 584 & 22.00 & 0.092 & ND \\
\hline $\begin{array}{l}\text { Evening (At } \\
6 \mathrm{pm} \text { ) }\end{array}$ & 34101 & 38368 & 11921 & 953 & 607 & 1697 & 13.00 & 0.182 & 1.00 \\
\hline
\end{tabular}

Table 16 for Wethedral/MCC junction

\begin{tabular}{|c|c|c|c|c|c|c|c|c|c|}
\hline \multirow[t]{2}{*}{ Period } & \multicolumn{6}{|c|}{ Cumulative Vehicle Types } & \multicolumn{3}{|c|}{ Pollutants } \\
\hline & $\begin{array}{c}\text { Sal. Veh. } \\
V_{1}\end{array}$ & $\begin{array}{l}\text { Wagon } \\
\text { Veh. } V_{2}\end{array}$ & $\begin{array}{c}\text { Mini } \\
\text { Buses } V_{3}\end{array}$ & $\begin{array}{c}\text { Coaster } \\
\text { Buses V V }\end{array}$ & $\begin{array}{c}\text { Big } \\
\text { Buses } V_{5}\end{array}$ & $\begin{array}{c}\text { Trucks } \\
V_{6}\end{array}$ & $\begin{array}{c}\text { CO } \\
\text { Ppm }\end{array}$ & $\begin{array}{l}\mathrm{NO}_{2} \\
\mathrm{Ppm}\end{array}$ & $\begin{array}{l}\mathrm{CH}_{4} \\
\mathrm{Ppm}\end{array}$ \\
\hline $\begin{array}{l}\text { Morning } \\
\text { (At 7am) }\end{array}$ & 1108 & 2353 & 112 & 18 & 24 & 86 & 22.00 & 0.075 & ND \\
\hline $\begin{array}{l}\text { Afternoon } \\
\text { (At 2pm) }\end{array}$ & 16151 & 15702 & 379 & 62 & 85 & 316 & 17.00 & 0.084 & ND \\
\hline $\begin{array}{l}\text { Evening } \\
\text { (At 6pm) }\end{array}$ & 30647 & 23098 & 883 & 164 & 195 & 633 & 21.00 & 0.126 & ND \\
\hline
\end{tabular}

ND: NON-DETECTED; NS: NOT STATED

\subsection{Commuters Traversing The Junctions}

The total commuters traversing the survey locations is the product of the number of vehicles and their weighted carrying capacities. From field observations the vehicles were averagely $60 \%$ loaded such that the following weighted capacities are derived.

Table 17 Passenger carrying capacity of vehicles

\begin{tabular}{|l|c|c|}
\hline Vehicle Type & Design Capacity & Weighted Capacity \\
\hline Saloon Vehicles & 5 & 3 \\
\hline Wagon Vehicles & 8 & 5 \\
\hline Mini Buses & 15 & 9 \\
\hline Coaster Buses & 33 & 20 \\
\hline Big Buses & 53 & 32 \\
\hline
\end{tabular}

Using the weighted carrying capacities and the cumulative vehicles accessing the locations, the total commuters traversing the locations are calculated as shown in Tables 3.18, 3.19, 3.20, 3.21 and 3.22 respectively. 
Table 18 Total Commuters Traversing IMSU Junction in a 12-Hour Day

\begin{tabular}{|c|c|c|c|}
\hline Veh. Types & Cumulative No & Weighted Capacity & Total Commuters \\
\hline Saloon Veh. $V_{1}$ & 25022 & 3 & 75,066 \\
\hline Wagon Veh. $V_{2}$ & 20813 & 5 & 104,065 \\
\hline Mini Buses $V_{3}$ & 4820 & 9 & 43,380 \\
\hline Coaster Buses $V_{4}$ & 357 & 20 & 7,140 \\
\hline Big Buses $V_{5}$ & 106 & 32 & 3,392 \\
\hline \multicolumn{3}{|c|}{ Total 51,118 } & 233043 \\
\hline
\end{tabular}

Table 19 Total Commuters Traversing Amakohia Junction in a 12-Hour Day

\begin{tabular}{|l|c|c|c|}
\hline Veh. Types & Cumulative No & Weighted Capacity & Total Commuters \\
\hline Saloon Veh. $V_{1}$ & 1621 & 3 & 4863 \\
\hline Wagon Veh. $V_{2}$ & 1531 & 5 & 7655 \\
\hline Mini Buses $V_{3}$ & 5526 & 9 & 49734 \\
\hline Coaster Buses $V_{4}$ & 645 & 20 & 12900 \\
\hline Big Buses $V_{5}$ & 127 & 32 & 4064 \\
\hline & Total 9450 & & 79,216 \\
\hline
\end{tabular}

Table 20 Total Commuters Traversing Assumpta Junction in a 12-Hour Day

\begin{tabular}{|l|c|c|c|}
\hline Veh. Types & Cumulative No & Weighted Capacity & Total Commuters \\
\hline Saloon Veh. $V_{1}$ & 20429 & 3 & 61,287 \\
\hline Wagon Veh. $V_{2}$ & 18853 & 5 & 94,245 \\
\hline Mini Buses $V_{3}$ & 3819 & 9 & 34,371 \\
\hline Coaster Buses $V_{4}$ & 382 & 20 & 7,640 \\
\hline Big Buses $V_{5}$ & 357 & 32 & 11,424 \\
\hline \multicolumn{3}{|c|}{ Total 43,840} & 208,987 \\
\hline
\end{tabular}

Table 21 Total Commuters Traversing Emmanuel College Junction in a 12-Hour Day

\begin{tabular}{|l|c|c|c|}
\hline Veh. Types & Cumulative No & Weighted Capacity & Total Commuters \\
\hline Saloon Veh. $V_{1}$ & 34101 & 3 & 102303 \\
\hline Wagon Veh. $V_{2}$ & 38368 & 5 & 191,040 \\
\hline Mini Buses $V_{3}$ & 11921 & 9 & 107,289 \\
\hline Coaster Buses $V_{4}$ & 953 & 20 & 19,060 \\
\hline Big Buses $V_{5}$ & 607 & 32 & 19,424 \\
\hline & Total 85,950 & 439,916 \\
\hline
\end{tabular}


Table 22 Total Commuters Traversing Wethedral/MCC Junction in a 12-Hour Day

\begin{tabular}{|l|c|c|c|}
\hline Veh. Types & Cumulative No & Weighted Capacity & Total Commuters \\
\hline Saloon Veh. $V_{1}$ & 30647 & 3 & 91,941 \\
\hline Wagon Veh. $V_{2}$ & 23098 & 5 & 115,490 \\
\hline Mini Buses $V_{3}$ & 883 & 9 & 7,947 \\
\hline Coaster Buses $V_{4}$ & 164 & 20 & 3,280 \\
\hline Big Buses $V_{5}$ & 195 & 32 & 6,240 \\
\hline \multicolumn{3}{|c|}{ Total 54,987} & 224,898 \\
\hline
\end{tabular}

Of the five junctions investigated Emmanuel College Junction recorded the highest number of vehicles and commuters traversing and highest values of pollutants. Further studies are based on the results from here, as a worst case scenario.

Table 23 shows the amount of the three pollutants of interest emitted into the environment by the vehicles traversing the Emmanuel college junction within 12 hours of the day.

Table 23 Total Pollutants Contribution by Vehicles that Accessed Emmanuel College Junction

\begin{tabular}{|c|c|c|c|c|c|c|c|}
\hline \multirow[t]{2}{*}{ Veh. Types } & \multirow{2}{*}{$\begin{array}{l}\text { Total } \\
\text { Veh. }\end{array}$} & \multicolumn{2}{|c|}{ Carbon Monoxide } & \multicolumn{2}{|c|}{ Nitrogen Dioxide } & \multicolumn{2}{|c|}{ Methane } \\
\hline & & Rate & $\begin{array}{c}\text { Total } \\
\times 10^{3} \text { ppm }\end{array}$ & Rate & $\begin{array}{c}\text { Total } \\
\times 10^{3} \mathrm{ppm}\end{array}$ & Rate & $\begin{array}{c}\text { Total } \\
\times 10^{6} \mathrm{ppm}\end{array}$ \\
\hline Saloon Cars $V_{1}$ & 34101 & 234.3 & 7989.86 & 0.49 & 16.71 & 422.9 & 144.21 \\
\hline Wagon Veh.V2 & 38368 & 158.97 & 6099.36 & 0.49 & 18.80 & 81.67 & 3.13 \\
\hline Mini Buses $V_{3}$ & 11921 & 225.93 & 2693.31 & 0.22 & 2.622 & 2923.33 & 34.85 \\
\hline Coaster Buses $V_{4}$ & 953 & 234.30 & 223.29 & 0.63 & 0.6 & 439.67 & 0.42 \\
\hline Big Buses $V_{5}$ & 607 & 42 & 5.49 & 5.37 & 3.26 & 12.67 & 0.0077 \\
\hline Total & 85,950 & & $\begin{array}{c}17,031.29 \mathrm{x} \\
10^{3} \mathrm{ppm}\end{array}$ & & $\begin{array}{c}41.992 \\
\times 10^{3} \mathrm{ppm}\end{array}$ & & $\begin{array}{c}182.62 \\
\times 10^{6} \mathrm{ppm}\end{array}$ \\
\hline
\end{tabular}

\subsection{Model Development}

The aim of the model is to achieve a remix of the vehicles such that the total commuters of 439,916 are served with a reduction in number of vehicles and pollutants released into the environment. This can be obtained by optimizing the model equations obtained.

Solving the Objective function obtained from table 4.21;

$$
3 v_{1}+5 v_{2}+9 v_{3}+20 v_{4}+32 v_{5}=439916 \quad \text { Eqn (1) }
$$

Subject to:

For a $40 \%$ reduction in $\mathrm{CO}$,

$$
234.3 v_{1}+158.97 v_{2}+225.93 v_{3}+234.3 v_{4}+42 v_{5}=10,218.774 \times 10^{3} \text { Eqn (2) }
$$

For a $50 \%$ reduction in $\mathrm{NO}_{2}$,

$$
0.49 v_{1}+0.49 v_{2}+0.22 v_{3}+0.63 v_{4}+5.51 v_{5}=20.996 \times 10^{3} \quad \text { Eqn (3) }
$$


For a $50 \%$ reduction in $\mathrm{CH}_{4}$,

$$
4229 v_{1}+81.67 v_{2}+2923.33 v_{3}+439.67 v_{4}+12.67 v_{5}=91.31 \times 10^{6} \quad \text { Eqn (4) }
$$

That $80 \%$ of the commuters use buses

$$
0+0+9 v_{3}+20 v_{4}+32 v_{5}=351,933
$$

It should be noted that the ambient pollutants measured indicated that a reduction by about half of the emission will bring them within the acceptable standards; Tables 4.12 to 4.16

In Matrix form the five equations are thus;

\begin{tabular}{|l|l|l|l|l|l|}
\hline $\mathbf{V}_{\mathbf{1}}$ & $\mathbf{V}_{\mathbf{2}}$ & $\mathbf{V}_{\mathbf{3}}$ & $\mathbf{V}_{\mathbf{4}}$ & $\mathbf{V}_{\mathbf{5}}$ & \\
\hline 3 & 5 & 9 & 20 & 32 & 439,916 \\
\hline 234.3 & 158.97 & 225.93 & 234.3 & 42 & $10,218,774$ \\
\hline 0.49 & 0.49 & 0.22 & 0.63 & 5.51 & 20,996 \\
\hline 4229 & 81.67 & 2923.33 & 439.67 & 12.67 & $91,310,000$ \\
\hline 0 & 0 & 9 & 20 & 32 & 351,933 \\
\hline
\end{tabular}

Solving the matrix using Tora Equation Solver yields;

$V_{1}=4,278.05, V_{2}=15,029.77, V_{3}=23,688.8, V_{4}=6,220.14, V_{5}=447.85$

Using these values (rounded off), multiplied by the weighted capacity of each vehicle type yields Table 24 while the values multiplied by the rate of emission of each vehicle type yields Table 25 .

Table 24 Total Commuters Provided For By The New Scheme

\begin{tabular}{|l|c|c|c|}
\hline \multicolumn{1}{|c|}{ Veh. Types } & Number of Vehicle & Weighted Capacity & Total \\
\hline Saloon Veh. $\mathrm{V}_{1}$ & 4,278 & 3 & 12,834 \\
\hline Wagon Veh. $\mathrm{V}_{2}$ & 15,050 & 5 & 75,150 \\
\hline Mini Buses $\mathrm{V}_{3}$ & 23,689 & 9 & 213,201 \\
\hline Coaster Buses $\mathrm{V}_{4}$ & 6,220 & 20 & 124,400 \\
\hline Big Buses $\mathrm{V}_{5}$ & 448 & 32 & 14,336 \\
\hline Total & 49,665 & & 439,921 \\
\hline
\end{tabular}

Table 24 shows a total of 49,665 vehicles for the cumulative commuters of 439,921 as against 85,950 vehicles for 439,916 commuters in Table 21 . This amounts to a reduction of 36,285 vehicles or $42.22 \%$ in passenger traffic volume.

Table 25 shows exhaust emissions of 10,219,880 ppm of Carbon monoxide, 20,998ppm of Nitrogen dioxide and 91,312,000ppm of Methane as against 17,005,800ppm, 41,992ppm and 182,620,000ppm respectively in Table 4.23. These amount to $40 \%$ reduction in Carbon monoxide, $50 \%$ reduction in Nitrogen dioxide and $50 \%$ reduction in Methane, of exhaust emissions due to passenger vehicles.

The analyses of the field data revealed a typical existing passenger vehicle mix of 34,101:38,368: 11,921: 955: 607, totaling 85,950 for Salon Vehicles: Wagon vehicles: Mini Buses: Coaster Buses: Big Buses, for a total of 439,916 commuters, from Table 21 
The ratio can therefore be written as $56.2: 63.7: 19.6: 1.6: 1$

It also shows a very high volume of 72,469 vehicles out of 85,950 or $84.3 \%$ of low-passenger carrying capacity vehicles (salons and wagons).

These low-passenger carrying vehicles emit into the environment,

$14,089.22 \times 10^{3} \mathrm{ppm}$ or $82.7 \%$ of the Carbon Monoxide

$35.51 \times 10^{3} \mathrm{ppm}$ or $84.6 \%$ of the Nitrogen dioxide and $147.34 \times 10^{6} \mathrm{ppm}$ or $80.7 \%$ of the Methane.

Table 25 Total Pollutants Emission by the New Scheme

\begin{tabular}{|c|c|c|c|c|c|c|c|}
\hline \multirow[t]{2}{*}{ Veh. Type } & \multirow{2}{*}{$\begin{array}{c}\text { Total } \\
\text { No. }\end{array}$} & \multicolumn{2}{|c|}{ Carbon Monoxide } & \multicolumn{2}{|c|}{ Nitrogen Dioxide } & \multicolumn{2}{|c|}{ Methane } \\
\hline & & Rate & $\begin{array}{l}\text { Total } \\
\times 10^{3}\end{array}$ & Rate & $\begin{array}{l}\text { Total } \\
\times 10^{3}\end{array}$ & Rate & $\begin{array}{l}\text { Total } \\
\times 10^{6}\end{array}$ \\
\hline Saloon Cars $V_{1}$ & 4,278 & 234.3 & 1002.335 & 0.49 & 2.096 & 4229 & 18.092 \\
\hline Wagon Veh. $V_{2}$ & 15,030 & 158.97 & 2389.32 & 0.49 & 7.365 & 81.67 & 1.228 \\
\hline Mini Buses $V_{3}$ & 23,689 & 225.93 & $5,352.056$ & 0.22 & 5.212 & 2923.33 & 69.251 \\
\hline Coaster Buses $V_{4}$ & 6,220 & 234.30 & $1,457.35$ & 0.63 & 3.919 & 439.67 & 69.251 \\
\hline Big Buses $V_{5}$ & 448 & 42 & 18.816 & 5.37 & 2.406 & 12.67 & 2.735 \\
\hline Total & 49,665 & & $\begin{array}{l}10,219.88 \\
X 10^{3}\end{array}$ & & $\begin{array}{l}20.998 \\
X 10^{3}\end{array}$ & & $\begin{array}{l}91.312 \\
\times 10^{6}\end{array}$ \\
\hline
\end{tabular}

This scenario of many vehicles plying the roads, carrying a few passengers but releasing high pollutants resulting in the twin problems of traffic congestion on the roads and highly polluting the environment is very typical in Nigerian Cities. It therefore needs to be addressed.

The new scheme being put forward has the following corresponding ratio as can be seen from Table 24 .

$4,278: 15,050: 23,689: 6,220: 448$ for 439,921 commuters.

This can be written as 9.5: 33.6: 52.9: 13.9: 1

It is now tilted to favour the high-passenger-carrying vehicles while taking into account pollutants release by them. In the new scheme, the low-passenger-carrying vehicles now total only 19,328 vehicles out of 49,665, amounting to $38.9 \%$.

In the new scheme, the low-passenger-carrying vehicles now emit to the atmosphere:

$3,391.66 \times 10^{3} \mathrm{ppm}$ or $33.2 \%$ Carbon monoxide

9. $461 \times 10^{3} \mathrm{ppm}$ or $45.1 \%$ of Carbon monoxide and

$19.32 \times 10^{6} \mathrm{ppm}$ or $21.2 \%$ of Methane.

Worthy of note in the new scheme is that vehicles of high-passenger-carrying capacities release greater percentage of the pollutants. In the typical case the buses with a combined capacity of 351,937 , which is $80 \%$ of total demand of 439,921 , will emit $66.8 \%$ of carbon monoxide, $54.9 \%$ of Nitrogen dioxide and $78.8 \%$ Methane.

Note also that the new scheme reduces total traffic volume by 36,285 vehicles from 85,950 to 49,665 representing $42.2 \%$, for a little more commuters. The new scheme as in Tables 3.25 will result in exhaust emissions of 10,219,880 ppm of Carbon monoxide, 20,998ppm of Nitrogen dioxide and 91,312,000ppm of Methane as against 17,005,800ppm, 
41,992ppm and 182,620,000ppm respectively in Table 23. These amount to $40 \%$ reduction in Carbon Monoxide, 50\% reduction in Nitrogen dioxide and 50\% reduction in Methane, of exhaust emissions due to passenger vehicles.

\section{Conclusion}

The existing commuter vehicle mix in Owerri, Imo State Nigeria is 56,2:63,7:19.6:1.6:1 for salons, wagons, mini buses, coaster buses and big buses respectively. This gives a total of 85,950 vehicles at a typical junction in a 12 hour period. The ambient air pollutants associated with this existing scenario is at a level well above the WHO acceptable standards.

By adopting the newly developed model, a remix of 10:33:53;14;1 of the same vehicle types is achieved. Thus, there is a reduction of about 40-50 percent in traffic volume and associated target air pollutants.

This is obviously more sustainable and more desirable in the $21^{\text {st }}$ century urban centre of Owerri.

\section{Recommendations}

This study has shown that mini-buses and coaster buses have advantage over salon cars, wagon vehicles and big buses in terms of traffic congestions and pollutants release into the environment. Thus, the two bus types could be said to have least pollutants release per passenger carried.

To achieve the desired remix, it is recommended that government should adopt economic policies embedded in some kind of "push and pull" strategy, leveraging on "disincentive and incentive" measures. Specifically government should;

- deliberately increase licensing fees for salons and wagons

- $\quad$ introduce bus lanes on major roads (Orlu, Okigwe, Wethedral, Asuumpta, Egbu, Douglas), which gives access to buses only thus reducing their trip time

- $\quad$ introduce equal toll fees on city roads for salons, wagon, and buses

- $\quad$ introduce annual parking fees for cars and wagons.

- encourage private-private or public-private cooperations in the public bus transportation system with grants.

- consider a regulatory policy of outright ban of such low-carrying-capacity vehicles like salons and wagons for commercial purposes, particularly on some major roads where bus lanes have been recommended.

\section{Compliance with ethical standards}

\section{Disclosure of conflict of interest}

No conflict of interest.

\section{References}

[1] Aravelli Apaina. Multi-objective Design Optimization of Engineering Systems: Uncertainty Approach and Practical Applications, Open Access dissertation ASTM International. 2014.

[2] Brundtland Commission. (World Commission on Environmental and Development), Our Common Future, Oxford University Press, Central Pollution Con troll Board (2010), study of the Exhaust Gases from different fuel based vehicles for carbonyls and Methane Emission; Control of Urban Pollution Series. 1987.

[3] Drumheller B, Quaid A, Wyman M, Liljenwall J, Young A. Sustainable Transportation Options for protecting the climate. International Council for Local Environmental Initiatives: Berkeley. 2001.

[4] Encyclopaedia Britanica. Owerri, Nigeria Retrieved from hhtps://www.britanica.com/place/owerri. 2019.

[5] https://www.wisdomjobs.com Solving LP Problems Using Computer with TORA-Quantitative Technology for Management.

[6] Merkisz-Guaranowska AJ, Merkisz M, Kozak M Jacyna. WTT Transaction on the Built Environment, vol. 130. WTT Press. 2013. 
[7] Murtaza H, Eric M. Effects of Transportation Infrastructure and Location on Residential Real Estate Values: Application of Spatial Autoregressive Techniques; Transportation Research Record Journal of the Transportation Research Board.

[8] Oxford Advance Learners Dictionary.

[9] Richards J. Play Time for Old Tyres, Swindon Engineering and Physical Sciences Research Council. 1999.

[10] Wikipedia. Mode of Transport https://en.m.wikipedia.org/wiki. 2018.

[11] Wikipedia. Air Pollution https.//en.wikipedia.org/wiki/Air_pollution. 2020.

[12] World Health Organisation. strategies for Healthy sustainable transportation; https://www.who:nd.transport.strategies. 2020. 